\title{
Report of the BIPM/IAU Joint Committee on Relativity for Space-Time Reference Systems and Metrology
}

\author{
G. Petit \\ Chairman, BIPM/IAU Joint Committee on relativity \\ Bureau International des Poids et Mesures 92312 Sèvres Cedex France
}

\begin{abstract}
The BIPM/IAU Joint Committee on relativity for spacetime reference systems and metrology (JCR) has worked in collaboration with the IAU Working Group on relativity for celestial mechanics and astrometry (RCMA) on the problems of astronomical relativistic space-time reference frames and the RCMA has proposed to the IAU a consistent framework for defining the barycentric and geocentric celestial reference systems at the first post-Newtonian level. Because new clock technology and space missions will necessitate the application of this framework for time and frequency measurements in the solar system, practical resolutions are proposed by the JCR to the IAU to allow a consistent treatment of these measurements. The paper outlines the conclusions of the work which resulted in the proposed IAU resolutions.
\end{abstract}

\section{Introduction}

In its 1991 General Assembly the International Astronomical Union (IAU) explicitly adopted the general theory of relativity as the theoretical framework for the definition and realization of space-time reference frames (IAU, 1991). Barycentric and geocentric coordinate time scales and the relativistic transformations between them were defined, together with procedures for their realization.

Previous work in the CCDS Working Group on the application of general relativity to metrology (Guinot, 1997 and references therein) has explicitly applied the IAU 1991 framework to time metrology. When studying the consequences of the increasing accuracy in the realization of the SI units, and particularly of the SI second, it recognized that the realized and expected progresses in time metrology were such as to require an extension of the IAU 1991 framework. Based on these conclusions and recognizing that, in this context, metrology could not be separated from the definition and realization of space-time reference systems, the BIPM proposed to the IAU to create a Joint Committee to study these matters.

At the Kyoto General Assembly (1997), the IAU endorsed, by its Resolution B3 (1997), the creation of the Joint Committee on Relativity for space-time reference systems and metrology (JCR). Also the IAU Working Group on Relativity in Celestial Mechanics and Astrometry (RCMA) continued under the chairmanship of M. Soffel and a common document was established: a "List of issues" (document available on the web: www.bipm.fr/WG/CCTF/JCR/jcrissue.html) 
discussing as much as possible all topics that may be addressed by the two working bodies. Circulars describing the work of the JCR are issued as required and the web pages of the JCR (ww. bipm.fr/WG/CCTF/JCR) are updated with each Circular.

At an early stage, some discussions took place on whether the formalism should be based strictly on the General Relativity theory or on some parametrized theory. Although no complete consensus could be reached at that time, the detailed work started with General Relativity on the basis that it was more likely to reach a final stage, i.e. a form that can be adopted as a Recommendation to extend the IAU 1991 framework. The RCMA initiated a theoretical document aiming at describing completely the reference systems, the coordinate transformations, and the equations of motion in the solar system. The JCR initiated studies on the shortcomings in the definition and usage of the IAU 1991 framework as well as on the application of the extended framework, with particular emphasis on time and frequency, time transformations and the realization of coordinate times.

In section 2, we recall the content of the IAU 1991 resolution A4 dealing with General Relativity and expose some of the limitations of this framework. The practical case of the extension of the IAU 1991 framework for time and frequency applications in the solar system is discussed in section 3, which forms the basis for the new resolutions to be proposed to the IAU and other concerned bodies.

\section{The IAU 1991 framework}

\subsection{The IAU 1991 recommendations}

The IAU resolution A4(1991) contains nine recommendations, the first five of which are directly relevant to our discussion.

In the first recommendation, the metric tensor for space-time coordinate systems $(t, \mathbf{x})$ centered at the barycenter of an ensemble of masses is recommended in the form

$$
\begin{aligned}
& g_{00}=-1+\frac{2 U(t, \mathbf{x})}{c^{2}}+\mathcal{O}\left(c^{-4}\right), \\
& g_{0 i}=\mathcal{O}\left(c^{-3}\right), \\
& g_{i j}=\delta_{i j}\left(1+\frac{2 U(t, \mathbf{x})}{c^{2}}\right)+\mathcal{O}\left(c^{-4}\right) .
\end{aligned}
$$

where $c$ is the speed of light in vacuum $(c=299792458 \mathrm{~m} / \mathrm{s}), U$ is the sum of the gravitational potentials of the ensemble of masses and of a tidal potential generated by bodies external to the ensemble, the latter potential vanishing at the barycenter. The algebraic sign of $U$ is taken to be positive. This recommendation recognizes that space-time cannot be described by a single coordinate system. The recommended form of the metric tensor can be used not only to describe the barycentric reference system of the whole solar system resulting in 
the BRS, but also to define the geocentric reference system (GRS) centered in the center of mass of the Earth with a suitable function $U$, now depending upon geocentric coordinates. In analogy to the GRS a corresponding reference system can be constructed for any other body of the solar system.

In the second recommendation, the origin and orientation of the space coordinate grids for the solar system (BRS) and for the Earth (GRS) are defined. Notably it is specified that the space coordinate grids of these systems should show no global rotation with respect to a set of distant extragalactic objects. It also specifies that the SI (International System of Units) second and the SI meter should be the physical units of proper time and proper length in all coordinate systems. It states in addition that the time coordinates should be derived from an Earth atomic time scale.

The third recommendation defines $T C B$ (Barycentric Coordinate Time) and TCG (Geocentric Coordinate Time) - the time coordinates of the BRS and GRS, respectively. The recommendation also defines the origin of the times scales (their reading on 1977 January $1,0^{\mathrm{h}} 0^{\mathrm{m}} 0^{\mathrm{s}} T A I(J D=2443144.5 T A I)$ must be 1977 January $1,0^{\mathrm{h}} 0^{\mathrm{m}} 32.184^{\mathrm{s}}$ ) and declares that the units of measurements of the coordinate times of all reference systems must coincide with the SI second and SI meter. The relationship between $T C B$ and TCG is given by a full 4-dimensional transformation

$$
T C B-T C G=c^{-2}\left[\int_{t_{0}}^{t}\left(\frac{v_{E}^{2}}{2}+U_{e x t}\left(t, \mathbf{x}_{E}(t)\right)\right) d t+v_{E}^{i} r_{E}^{i}\right]+\mathcal{O}\left(c^{-4}\right),
$$

where $x_{E}^{i}$ and $v_{E}^{i}$ are the barycentric coordinate position and velocity of the geocenter, $r_{E}^{i}=x^{i}-x_{E}^{i}$ with $x^{i}$ the barycentric position of the observer, and $U_{\text {ext }}\left(t, \mathbf{x}_{E}(t)\right)$ is the Newtonian potential of all solar system bodies apart from the Earth evaluated at the geocenter.

In the fourth recommendation another time coordinate, Terrestrial Time $(T T)$, is defined for the GRS. It differs from $T C G$ by a constant rate only

$$
T C G-T T=L_{G} \times(J D-2443144.5) \times 86400, \quad L_{G} \approx 6.969291 \times 10^{-10},
$$

so that the unit of measurement of $T T$ agrees with the SI second on the geoid. $T T$ represents an ideal form of $T A I$, the divergence between them being a consequence of the physical defects of atomic clocks. The fifth recommendation states that the former barycentric time TDB may still be used where discontinuity with previous work is deemed to be undesirable.

\subsection{Some problems in the framework of the IAU 1991 recommen- dations}

Because of the form of the metric (1) in the IAU 1991 framework, time transformations and the realization of coordinate times in the barycentric system are not specified at the $c^{-4}$ level, i.e. at a level of a few parts in $10^{16}$ in rate. Within that approximation, constants $L_{B}$ and $L_{C}$ were introduced in notes to the IAU 1991 Recommendation 3 to express the mean rates between time scales as 


$$
\begin{aligned}
T C B-T D B= & L_{B} \times(J D-2443144.5) \times 86400 \\
& L_{B} \approx 1.550505 \times 10^{-8}, \text { and } \\
T C B-T C G= & L_{C} \times(J D-2443144.5) \times 86400+v_{E}^{i} r_{E}^{i} / c^{2}+P, \\
& L_{C} \approx 1.480813 \times 10^{-8},
\end{aligned}
$$

where $P$ represents periodic terms. Since JD is not specified to be a particular time scale, these constants were not properly defined so that confusion appeared in their usage. Following Fukushima (1995) we propose to use as more rigorous definitions, in order to overcome the above problems $\langle T C G / T C B\rangle=1-L_{C}$ and $\langle T T / T C B\rangle=1-L_{B}$, where $<>$ means a sufficiently long term average taken at the geocenter. The actual computation of $L_{C}$ and $L_{B}$ requires the integration of solar system ephemerides and the specification of an averaging duration. This process may be applied to the utmost accuracy, after a choice of ephemerides and averaging duration. For example Irwin and Fukushima (1999) determined $L_{C}=1.48082686741 \times 10^{-8} \pm 2 . \times 10^{-17}$. However, because no unambiguous definition may be provided for $L_{B}$ and $L_{C}$, these constants should not be used in formulating time transformations when it would require knowing their value with an uncertainty of order $1 \times 10^{-16}$ or less.

Another problem arising from the situation before IAU 1991 is that the barycentric dynamical time TDB did not have a good definition. This could be corrected by turning a specific value of $L_{B}$ into a defining constant thus providing in retrospect a good definition of TDB. It was not felt necessary to address explicitly this point in a recommendation.

The most important questions, however, concern the extension of the framework, at an uncertainty level beyond that covered by the 1991 formulation. This means to apply the extended post-Newtonian metric (Soffel, 2000) to the realization of the barycentric coordinate time TCB and to the time transformations at an adequate level of uncertainty, and is described in section 3.1. Finally, some consequences of the application of the IAU 1991 framework in the geocentric system will be considered in section 3.2.

\section{Time and frequency applications in the solar system: basis for proposed Resolutions}

For practical applications concerning time and frequency measurements in the solar system, it is necessary to consider a conventional model for the realization of time coordinates and time transformations. This model should be chosen so that i) its accuracy is significantly better than the expected performance of clocks and time transfer techniques, ii) it is consistent with the general framework of (Soffel, 2000) and iii) it may readily be used with existing astrometric quantities, e.g. solar systems ephemerides.

Regarding item (i), we may derive reasonable accuracy limits in a straightforward way. At present the best accuracies are reached by Cs-fountain clocks at less than two parts in $10^{15}$ in fractional frequency (Simon et al., 1998), with a frequency stability characterized by a standard Allan deviation of or$\operatorname{der} \sigma_{y}(\tau)=4 \times 10^{-14} \tau^{-1 / 2}$, for an integration time $\tau$ in seconds. In the near 
future, high accuracy laser cooled Rb clocks (Bize et al., 1999) and space-borne Cs clocks (Salomon et al., 1999) are expected to reach accuracies of a few parts in $10^{17}$ in fractional frequency and stabilities of order $\sigma_{y}(\tau)=1 \times 10^{-14} \tau^{-1 / 2}$, with $\tau$ in seconds. The uncertainty in the time transformations should induce errors that are always lower than the expected performance of these future clocks. Including a factor 2 as safety margin, we therefore conclude that time coordinates and time transformations should be realized with an uncertainty not larger than $5 \times 10^{-18}$ in rate or, for quasi-periodic terms, not larger than $5 \times 10^{-18}$ in rate amplitude and $0.2 \mathrm{ps}$ in phase amplitude.

For the spatial domain of validity of the transformations, we note that projects like SORT (Solar Orbit Relativity Test) plan to fly high-accuracy clocks to within $0.25 \mathrm{AU}$ of the Sun, but it turns out that formulations valid there will also be valid at a few solar radii from the Sun which is therefore the lower limit for the distance to the barycenter that we will consider. In the geocentric system we will consider locations from the Earth's surface up to geostationary orbits $(|\mathbf{X}|<50000 \mathrm{~km})$.

To comply with item (ii), we explicitly derive the developments in the next sub-section from the general framework presented in the RCMA report (Soffel, 2000 ), using the same conventions and notations. Regarding item (iii) we show below how the time transformations, e.g., TCB - TCG, may be performed with the existing astrometric quantities and tools.

\subsection{Barycentric celestial reference system}

Following (Soffel 2000), the metric tensor in the BCRS (this is the new name of the BRS, where $\mathrm{C}$ stands for Celestial) is expressed as

$$
\begin{aligned}
& g_{00}=-\left(1-\frac{2}{c^{2}}\left(w_{0}(t, \mathbf{x})+w_{L}(t, \mathbf{x})\right)+\frac{2}{c^{4}}\left(w_{0}^{2}(t, \mathbf{x})+\Delta(t, \mathbf{x})\right)\right), \\
& g_{0 i}=-\frac{4}{c^{3}} w_{i}(t, \mathbf{x}), \\
& g_{i j}=\left(1+\frac{2 w_{0}(t, \mathbf{x})}{c^{2}}\right) \delta_{i j},
\end{aligned}
$$

where $(t \equiv T C B, \mathbf{x})$ are the barycentric coordinates, $w_{0}=G \sum_{A} M_{A} / r_{A}$, with the summation carried out over all solar system bodies $A, \mathbf{r}_{A}=\mathbf{x}-\mathbf{x}_{A}, r_{A}=\left|\mathbf{r}_{A}\right|$, and where $w_{L}$ contains the expansion in terms of multipole moments, as defined in (Soffel, 2000), required for each body. The values of masses and multipole moments to be used may be found in IAU or IERS documents (IERS 1996), but care must be taken that the values are in SI units (not in so-called TDB units or TT units). The vector potential $w^{i}(t, \mathbf{x})=\sum_{A} w_{A}^{i}(t, \mathbf{x})$ and the function $\Delta(t, \mathbf{x})=\sum_{A} \Delta_{A}(t, \mathbf{x})$ are given later.

From (5) the transformation between proper time and $T C B$ may be derived. It reads:

$$
\mathrm{d} \tau / \mathrm{d} T C B=1-\frac{1}{c^{2}}\left(w_{0}+w_{L}+\frac{v^{2}}{2}\right)+\frac{1}{c^{4}}\left(-\frac{1}{8} v^{4}-\frac{3}{2} v^{2} w_{0}+4 v^{i} w^{i}+\frac{1}{2} w_{0}^{2}+\Delta\right) .
$$


To reach the uncertainty mentioned earlier, it is sufficient to express the vector potential $w_{A}^{i}(t, \mathbf{x})$ of body $A$ as

$$
w_{A}^{i}(t, \mathbf{x})=G\left[\frac{-\left(\mathbf{r}_{A} \times \mathbf{S}_{A}\right)^{i}}{2 r_{A}^{3}}+\frac{M_{A} v_{A}^{i}}{r_{A}}\right],
$$

where $\mathbf{S}_{A}$ is the total angular momentum and $v_{A}^{i}$ is the coordinate velocity of body $A$. As for the function $\Delta_{A}(t, \mathbf{x})$ it is sufficient to express it as

$$
\begin{aligned}
\Delta_{A}(t, \mathbf{x})= & \frac{G M_{A}}{r_{A}}\left[-2 v_{A}^{2}+\sum_{B \neq A} \frac{G M_{B}}{r_{B A}}+\frac{1}{2}\left(\frac{\left(r_{A}^{k} v_{A}^{k}\right)^{2}}{r_{A}^{2}}+r_{A}^{k} a_{A}^{k}\right)\right] \\
& +\frac{2 G v_{A}^{k}\left(\mathbf{r}_{A} \times \mathbf{S}_{A}\right)^{k}}{r_{A}^{3}}
\end{aligned}
$$

where $r_{B A}=\left|\mathbf{x}_{B}-\mathbf{x}_{A}\right|$ and $a_{A}^{k}$ is the coordinate acceleration of body $A$.

Evaluating the $\Delta_{A}$-terms for all bodies of the solar system, we find that $\left|\Delta_{A}(t, \mathbf{x})\right| / c^{4}$ may reach at most a few parts in $10^{17}$ in the vicinity of Jupiter and about $1 \times 10^{-17}$ close to the Earth. Presently, however, for all planets except the Earth, the magnitude of $\Delta_{A}(t, \mathbf{x}) / c^{4}$ in the vicinity of the planet is smaller than the uncertainty in $w_{0} / c^{2}$ or $w_{L} / c^{2}$ originating from the uncertainties in its multipole moments so that it is practically not needed to account for these terms. Nevertheless, when new astrometric observations allow us to derive the moments with adequate uncertainty, it will be necessary to do so. In any case, for the vicinity of a given body $A$, only the effect of $\Delta_{A}(t, \mathbf{x})$ is needed in practice, i.e., the effect of $\sum_{B \neq A} \Delta_{B}(t, \mathbf{x})$ is smaller than our accuracy specifications. For a clock in the vicinity of the Earth, to be compared with other clocks in the solar system or to $T C B$, it may thus be needed to account for $\Delta_{E}(t, \mathbf{x}) / c^{4}$. Also, in these formulas, the terms in $\mathbf{S}_{A}$ are needed to reach the mentioned uncertainty only for Jupiter $\left(S \approx 6.9 \times 10^{38} \mathrm{~m}^{2} \mathrm{~s}^{-1} \mathrm{~kg}\right)$ and Saturn $\left(S \approx 1.4 \times 10^{38} \mathrm{~m}^{2} \mathrm{~s}^{-1} \mathrm{~kg}\right)$, in the immediate vicinity of these planets.

Similarly, the transformation between $T C B$ and $T C G$ may be written as

$$
\begin{array}{r}
T C B-T C G=c^{-2}\left[\int_{t_{0}}^{t}\left(\frac{v_{E}^{2}}{2}+w_{0 e x t}\left(\mathbf{x}_{E}\right)\right) d t+v_{E}^{i} r_{E}^{i}\right] \\
-c^{-4}\left[\int_{t_{0}}^{t}\left(-\frac{1}{8} v_{E}^{4}-\frac{3}{2} v_{E}^{2} w_{0 e x t}\left(\mathbf{x}_{E}\right)+4 v_{E}^{i} w_{e x t}^{i}\left(\mathbf{x}_{E}\right)+\frac{1}{2} w_{0 e x t}^{2}\left(\mathbf{x}_{E}\right)\right) d t\right. \\
\left.-\left(3 w_{0 e x t}\left(\mathbf{x}_{E}\right)+v_{E}^{2} / 2\right) v_{E}^{i} r_{E}^{i}\right]
\end{array}
$$

where $t$ is $T C B$ and where the index ext refers to all bodies except the Earth. This equation is composed of terms evaluated at the geocenter (the two integrals) and of position dependent terms in $r_{E}$, with position dependent terms in higher powers of $r_{E}$ having been found to be negligible. The first integral may be computed from existing planetary ephemerides (Fukushima, 1995; Irwin and Fukushima, 1999). Since, in general, the planetary ephemerides are expressed in terms of a time argument $T_{e p h}$ which is close to $T D B$, rather than in terms of $T C B$, the first integral will be computed as 


$$
\int_{t_{0}}^{t}\left(\frac{v_{E}^{2}}{2}+w_{0 e x t}\left(\mathbf{x}_{E}\right)\right) d t=\left[\int_{t_{e p h_{0}}}^{t_{e p h}}\left(\frac{v_{E}^{2}}{2}+w_{0 e x t}\left(\mathbf{x}_{E}\right)\right) d t_{e p h}\right] /\left(1-L_{B}\right)
$$

Terms in the second integral of (9) are secular and quasi-periodic. They amount to $\sim 1.1 \times 10^{-16}$ in rate $(\mathrm{d} T C B / \mathrm{d} T C G)$ and primarily a yearly term of $\sim 30 \mathrm{ps}$ in amplitude (i.e. corresponding to periodic rate variations of amplitude $\sim 6 \times 10^{-18}$ ). Terms in $\Delta_{e x t}(t, \mathbf{x})$ that would appear in this integral are negligible in the vicinity of the Earth where this formula is to be used. Position dependent terms in $c^{-4}$ (the last two terms in (9)) are not negligible and reach, for example, an amplitude of $0.4 \mathrm{ps}\left(\sim 3 \times 10^{-17}\right.$ in rate $)$ in geostationary orbit.

The material in this sub-section is expressed in the Resolution 'Extended relativistic framework for time transformations and realization of coordinate times in the solar system' proposed to the IAU General Assembly in August 2000 .

\subsection{Geocentric reference system}

Evaluating the contributions of the higher order terms in (Soffel, 2000), it is found that the IAU 1991 framework with the metric of the form (1) is sufficient for time and frequency applications in the GRS in the light of present and foreseeable future clock accuracies. In particular a choice of gauge and of the state of rotation of the GRS (kinematically or dynamically non-rotating) does not influence the time and frequency application at the given level of accuracy. However, some care needs to be taken when evaluating the Earth's potential at the location of the clock which is not trivial when accuracy of order $10^{-18}$ is required (Klioner, 1992; Wolf and Petit, 1995; Petit and Wolf, 1996).

Presently, the time scale of reference for all practical matters on Earth is Terrestrial Time $T T$ or one of the scales realizing it and differing by some time offset (e.g., TAI,UTC,GPS-time). The definition of $T T$ reads: "time scale differing from the Geocentric Coordinate Time TCG by a constant rate, the unit of measurement of $T T$ being chosen so that it agrees with the SI second on the geoid." According to the transformation between proper and coordinate time, this constant rate is given by $\mathrm{d}(T T) / \mathrm{d}(T C G)=1-U_{g} / c^{2}=1-L_{G}$, where $U_{g}$ is the gravity (gravitational + rotational) potential on the geoid.

The gravity potential on the geoid $U_{g}$ is determined with an uncertainty slightly lower than $1 \mathrm{~m}^{2} \mathrm{~s}^{-2}$ (Groten, 1999) so that the corresponding rate uncertainty in the definition of $T T$ is of order $1 \times 10^{-17}$. In view of the performance of future clocks, it may be desirable to change the definition of $T T$ in a way that would ensure continuity but would not be limited by this uncertainty. One solution is to define $T T$ differing from $T C G$ by a fixed constant rate, i.e. $L_{G}$ becomes a defining constant. To ensure the best continuity, this conventional value of $L_{G}$ should be chosen as the current best estimation of $U_{g} / c^{2}$, i.e. $L_{G}=6.969290134 \times 10^{-10}$, from the value $U_{g}=62636856 \mathrm{~m}^{2} \mathrm{~s}^{-2}$ provided by the International Association of Geodesy Special Commission 3 (Groten, 1999).

The material in this sub-section is expressed in the Resolution 'Re-definition of Terrestrial Time TT' proposed to the IAU General Assembly in August 2000. 
Acknowledgments. The contributions of members of the JCR, which list may be found on the web site (www.bipm.fr/WG/CCTF/JCR/jcrsccor.html), as well as numerous other informal discussions, are gratefully acknowledged.

\section{References}

Bize, S. et al., 1999, Europhys. Lett., 45 (5), 558.

Fukushima, T., 1995, Astron. Astrophys., 294, 895.

Groten, E., 1999, Report of the IAG SC3 Fundamental Constants, XXII IAG General Assembly.

Guinot, B., 1997, Metrologia, 34, 261.

IAU, 1991, Transactions of the International Astronomical Union, XXIB (edited by J. Bergeron), Dordrecht, Kluwer.

IERS Conventions, 1996, International Earth Rotation Service Technical Note 21 (ed. by D.D.McCarthy), Observatoire de Paris.

Irwin, A. and Fukushima, T., 1999, Astron. Astrophys., 348, 642.

IUGG, 1991, Resolution 2.

Klioner, S.A., 1992, The problem of clock synchronization: a relativistic approach, Celestial Mechanics and Dynamical Astronomy, 53, 81.

Petit, G. and Wolf, P., 1996, IEEE Trans. IM 46, 2, 201.

Salomon, C. et al., 1999, Proc. $13^{\text {th }}$ EFTF meeting, in press.

Simon, E., Laurent, P., Clairon, A., 1998, Phys. Rev. A, 57 (1), 436.

Soffel, M., 2000, Proc. IAU Colloquium 180, this volume.

Wolf, P. and Petit, G., 1995, Astron. Astrophys., 304, 653. 